\title{
Magnetotransport in the CeIrIn 5 System: The Influence of Antiferromagnetic Fluctuations
}

\author{
Sunil Nair $\cdot$ M. Nicklas $\cdot$ J.L. Sarrao $\cdot$ J.D. Thompson • \\ F. Steglich · S. Wirth
}

Received: 16 October 2008 / Accepted: 20 October 2008 / Published online: 1 November 2008

(C) The Author(s) 2008. This article is published with open access at Springerlink.com

\begin{abstract}
We present an overview of magnetotransport measurements on the heavy-fermion superconductor CeIrIn 5 . Sensitive measurements of the Hall effect and magnetoresistance are used to elucidate the low-temperature phase diagram of this system. The normal-state magnetotransport is highly anomalous, and experimental signatures of a pseudogap-like precursor state to superconductivity, as well as evidence for two distinct scattering times governing the Hall effect and the MR, are observed. Our observations point out the influence of antiferromagnetic fluctuations on the magnetotransport in this class of materials. The implications of these findings, both in the context of unconventional superconductivity in heavy-fermion systems and in relation to the high-temperature superconducting cuprates, are discussed.
\end{abstract}

Keywords Heavy-fermion superconductors · Hall effect . Magnetoresistance

\section{Introduction}

Superconductivity - the zero electrical resistance state of matter-involves the formation of bound pairs of electrons, so-called Cooper pairs. The BCS theory [1] states that these electrons are bound together by dynamic lattice distortions (or phonons). This theory provides a remarkably accurate description for superconductivity in many, if not all,

S. Nair · M. Nicklas $\cdot$ F. Steglich $\cdot$ S. Wirth $(\bowtie)$

Max Planck Institute for Chemical Physics of Solids,

Noethnitzer Str. 40, 01187 Dresden, Germany

e-mail: wirth@cpfs.mpg.de

J.L. Sarrao · J.D. Thompson

Los Alamos National Laboratory, Los Alamos, NM 87545, USA "conventional" superconductors. Among the so-called "unconventional" superconductors, in particular two contrasting classes of materials - the heavy-fermion and the hightemperature cuprate superconductors-have posed an irrefutable challenge to this theory. The non-compliance with the standard BCS model is primarily based on the failure to reconcile how magnetism could coexist with the superconducting ground state in these systems. In conventional superconductors, it is known that tiny amounts of magnetic impurities rapidly destabilize the superconducting condensate. The aforementioned two families of superconductors, on the other hand, are comprised of dense arrays of magnetic ions. The observation of superconductivity in an inherently magnetic environment [2] has led researchers to postulate that (antiferro-)magnetic fluctuations themselves were the bosonic modes coupling the electrons into Cooper pairs, thus facilitating a superconducting ground state in these materials. The observation of superconductivity in the vicinity of antiferromagnetic quantum critical points (QCP, zerotemperature continuous phase transitions) in some heavyfermion systems [3] has lent further credence to this theory.

The contrast between these two families of materials is stark. Metals are typically well described by the LandauFermi liquid (LFL) theory. Although the heavy-fermion systems exhibit metallic behavior, this theory cannot be applied in certain cases, e.g. in the vicinity of a magnetic QCP that gives rise to an anomalous metallic state [4]. The parent high temperature superconductors, on the other hand, are Mott insulators: systems where strong electron-electron correlations promote an insulating ground state at the cost of a conducting one that would be expected from conventional band theories. In spite of this apparent difference, a surprising similarity in both the superconducting and normal-state properties of some heavy-fermion systems with the high- $T_{\mathrm{C}}$ 
cuprates has been observed. This has been brought to attention by recent observations in the $\mathrm{Ce} M \operatorname{In}_{5}(M=\mathrm{Co}$, Ir, Rh) family of compounds [5]. These quasi-two-dimensional systems have revealed a host of experimental signatures which are remarkably similar to those observed earlier in the high$T_{\mathrm{c}}$ cuprates [6]. For instance, the normal-state resistivity is seen to be linear as a function of temperature, the Hall effect is strongly temperature dependent, and an anomalous Nernst effect is observed above the superconducting upper critical field $H_{\mathrm{c} 2}$ [7]. The superconducting state is also unconventional, and it was suggested that the superconducting gap possibly has line nodes consistent with $d$ wave symmetry [8].

Here we summarize some of our recent results on the normal-state magnetotransport in the compound CeIrIn 5 . Using sensitive measurements of the Hall effect and magnetoresistance (MR), a comprehensive low-temperature phase diagram of this system is charted out. The implications of our observations are discussed, both in terms of discerning the influence of antiferromagnetic fluctuations on the magnetotransport and in placing these heavy-fermion superconductors in perspective to the superconducting cuprates.

\section{Experimental Details}

Measurements of the Hall effect and MR are conducted on a single crystalline sample of CeIrIn ${ }_{5}$ within the temperature range $0.05 \mathrm{~K} \leq T \leq 2.5 \mathrm{~K}$ in the form of isothermal field sweeps. The magnetic field (of up to $15 \mathrm{~T}$ ) is applied parallel to the crystallographic $c$ axis, and the Hall voltage is extracted as the asymmetric component under magnetic field reversal. Low-temperature transformers are used in conjunction with low-noise voltage preamplifiers to enable an effective resolution of better than $\pm 0.01 \mathrm{nV}$.

\section{Results and Discussions}

The system CeIrIn 5 , which is reported to have a resistive superconducting transition temperature of $1.2 \mathrm{~K}$ [9], is probably the most enigmatic one in the $\mathrm{Ce} M \mathrm{In}_{5}$ series of compounds: the superconductivity in this system seems to be separated from the magnetic instability in the field-temperature-pressure phase space. In the other primary members of this family (CeCoIn 5 and CeRhIn 5 ), it is known that superconductivity is closely tied to the suppression of magnetic order. For instance, in the ambient-pressure superconductor $\mathrm{CeCoIn}_{5}$, the magnetic instability is proposed to be in the vicinity of $H_{\mathrm{c} 2}[10]$. However, in CeIrIn 5 , the magnetic instability (which was suggested to be metamagnetic in origin) is speculated to lie above $25 \mathrm{~T}$, whereas $H_{\mathrm{c} 2}$ is only of the order of $3 \mathrm{~T}$ [11]. Though the range of our measurements does not encompass the magnetic instability, this
Fig. $1 H-T$ phase diagram of CeIrIn 5 as determined from a combination of Hall-effect and MR measurements

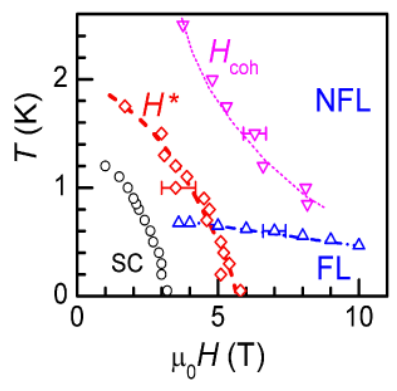

contrast is also clearly manifested in our data. In systems exhibiting a typical antiferromagnetic QCP (believed to be the case in $\mathrm{CeCoIn}_{5}$ ), an applied magnetic field destabilizes the antiferromagnetic order and facilitates the recovery of the LFL regime. In CeIrIn 5 , however, a reverse trend is observed wherein the applied magnetic field suppresses (and not enhances) the LFL region. Thus the bulk superconductivity in CeIrIn $_{5}$ appears to emerge from within the LFL region.

Figure 1 summarizes the phase diagram of $\mathrm{CeIrIn}_{5}$ as determined from our magnetotransport measurements [12]. Two distinguishable crossover lines in this phase diagram are clearly related to the magnetic instability at about $25 \mathrm{~T}$ : (i) The LFL to non-Fermi liquid (NFL) crossover, as determined by deviations from Kohler's scaling rule and (ii) the onset of a coherent Kondo scattering regime, as determined from a crossover in the sign of the MR at $H_{\text {coh }}$. The LFLNFL crossover is seen to be in good agreement with prior reports, and a linear extrapolation indeed intercepts the magnetic field axis at about $25 \mathrm{~T}$. Though the functional form of the coherent to incoherent Kondo regime is non-trivial, a crossing between these two lines is unlikely, and the Kondo coherence would also be expected to vanish at the magnetic instability.

A striking result from the analysis of our magnetotransport data pertains to the observation of a precursor state to superconductivity in CeIrIn 5 . This state, which is seen as a curve that envelops $H_{\mathrm{c} 2}$ in Fig. 1, was inferred from the field-dependence of the Hall angle $\theta_{H}$. Though rarely utilized as a means of investigating heavy-fermion systems, prior work in the superconducting cuprates has demonstrated that its cotangent $\cot \theta_{H}=\rho_{x x} / \rho_{x y}$ is a quantity of fundamental interest as it is primarily a measure of the charge carrier mobility. A quadratic temperature dependence of $\cot \theta_{H}$ was observed in the cuprates which appeared to be independent of the charge carrier density and the extent of impurity substitution [13]. Since the resistivity $\rho_{x x}$ is linear in $T$, this $T^{2}$ dependence of $\cot \theta_{H}$ is thought to be a manifestation of the fact that there are two distinct scattering rates which independently influence the resistivity and the Hall effect ( $\tau_{\text {tr }}$ and $\tau_{H}$, respectively). Moreover, deviations from the $T^{2}$ behavior of $\cot \theta_{H}$ were interpreted in terms of the onset of the pseudogap state in the cuprates [14]. Our measurement protocol (we conducted isothermal 
field sweeps) enables us to investigate the magnetic-field dependence of this quantity in detail, and $\cot \theta_{H}$ is seen to have an $H^{-1}$ dependence within a substantial region of the $H-T$ phase space. In the vicinity of the superconducting region, systematic deviations from this $H^{-1}$ dependence are seen (starting at a critical field $H^{*}$ ), the field and temperature dependence of which is exhibited in the Fig. 1. Our measurements clearly indicate the existence of a pseudogap-like precursor state to superconductivity in CeIrIn 5 , a phenomenon which may be generic to many other heavy-fermion superconductors as well. Interestingly, the critical field of this precursor state scales onto the superconducting upper critical field $H_{\mathrm{c} 2}$, implying that both of them might arise from the same underlying physical mechanism [12].

The presence of this precursor state appears to crucially influence the normal-state magnetotransport in CeIrIn 5 . Moreover, it seems to influence $\tau_{\text {tr }}$ and $\tau_{H}$ in a disparate fashion. This is clearly borne out by two key experimental observations: Firstly, contrary to prior reports [15], the modified Kohler's scaling (which relates the MR to the Hall angle) breaks down in the precursor state. Secondly, a model-dependent single parameter scaling of magnetotransport quantities - using the demarcation $H^{*}(T)$ of the precursor state-is seen to be applicable only for the Hall angle [16]. This is shown in Fig. 2, where the normalized Hall angle $\cot \theta_{H}(H) / \cot \theta_{H}\left(H^{*}\right)$ is plotted as a function of the normalized field $\left(H / H^{*}\right)$ revealing good scaling behavior. The fact that neither the resistivity nor the Hall effect individually exhibits this scaling implies that the precursor state preferentially influences the Hall channel. This scaling clearly demonstrates that the precursor state in CeIrIn ${ }_{5}$ represents a fundamental energy scale of the system; in addition to the well recognized energy scales corresponding to the crystal electric field, the intersite coupling and the singleion Kondo effect. It also re-emphasizes the presence of two distinct scattering times in these systems, in similarity to observations in the superconducting cuprates. Although the existence of two scattering times in heavy-fermion systems remains to be investigated in adequate detail, this scenario might well be applicable to other systems.

Our key observations, namely an $H^{-1}$ dependence of the Hall angle (which in turn is used to demarcate the precursor state) and two distinct scattering times, can be adequately described by two theories which have been extensively used earlier in the cuprates. The spin-charge-separation scenario developed by Anderson [17] postulates the formation of two different quasiparticles each of which is associated with the spin and charge degrees of freedom. The two different scattering times then correspond to dissimilar scattering events associated with these two different kinds of quasiparticles. On the other hand, the nearly antiferromagnetic Fermi liquid (NAFL) scenario [18] postulates the modification of scattering rates along different regions of the Fermi surface. This is

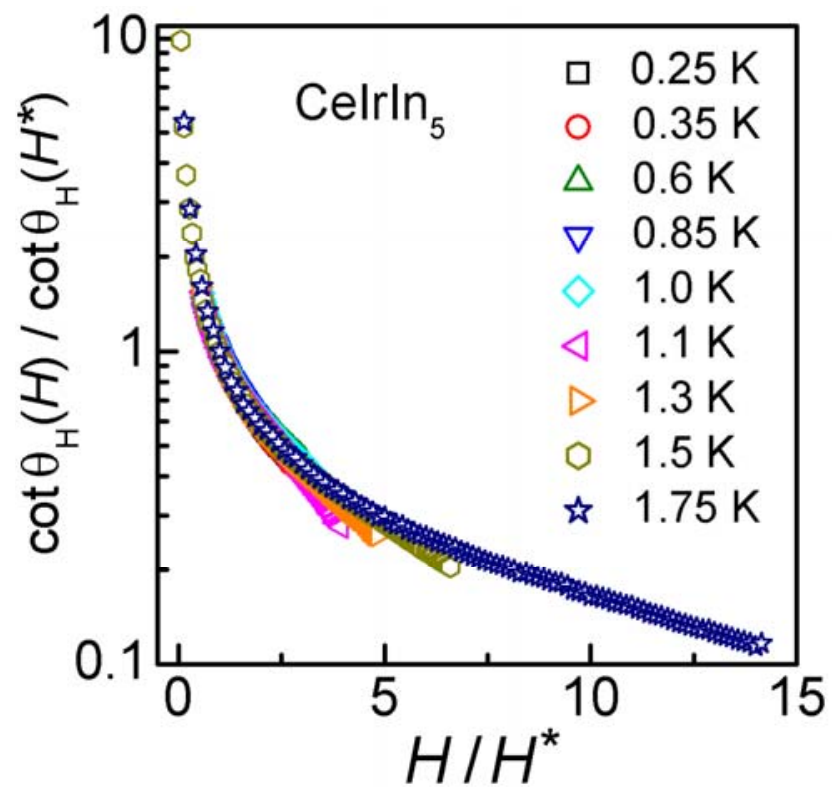

Fig. 2 Scaling of $\cot \theta_{H}$ and the field $H$ normalized with respect to the values at $H^{*}(T)$, which demarcates the precursor state. All the isotherms are seen to collapse on a single generic curve

accomplished by the formation of so-called hot spots which represent regions of the Fermi surface where it intersects the antiferromagnetic Brillouin zone and where scattering becomes singular. Thus, all the transport coefficients are renormalized with respect to the anisotropy associated with the different regions of the Fermi surface. Interestingly, both of these scenarios can explain the observed $\mathrm{H}^{-1}$ dependence of the Hall angle.

While the spin-charge-separation scenario cannot be ruled out, the NAFL picture is a particularly attractive one for the $\mathrm{Ce} M \mathrm{In}_{5}$ compounds. In $\mathrm{CeCoIn}_{5}$, for instance, an anisotropic destruction of the Fermi surface in the limit $T \rightarrow 0$ was inferred, analogously to the pseudogap phase in the cuprates [19]. It was suggested that this destruction of the Fermi surface could be caused by uniaxial spin fluctuations. Though the superconductivity in CeIrIn ${ }_{5}$ is reasonably separated from the magnetic instability in the $H-T$ phase space, the presence of antiferromagnetic fluctuations in the regime investigated here has also been inferred from Nuclear Quadrapole Resonance (NQR) measurements [20]. These fluctuations themselves are thought to be anisotropic, with the magnetic correlation length along the basal plane being substantially larger than the one along the $c$ axis. Thermal conductivity measurements have suggested that the superconducting gap in this system may have a $d_{x^{2}-y^{2}}$ symmetry, which is consistent with a scenario in which superconductivity is mediated by incipient antiferromagnetic fluctuations [21]. The fact that antiferromagnetic spin fluctuations are a crucial ingredient in determining the electronic ground state of these systems was also demonstrated by a chemical substitution study [22]: small amounts of Cd substitution on 
the In site shift the delicate balance between magnetism and superconductivity. The evolution of a magnetically ordered ground state with $\mathrm{Cd}$ substitution could not be accounted for by simple unit-cell volume considerations implying that the observed effects were a consequence of tailoring the electronic band structure.

A phenomenon in the cuprate superconductors, which had no apparent analog in the heavy-fermion systems, was the formation of charge stripes. Such stripes would allow for both antiferromagnetism (with localized spins) and superconductivity (with mobile charge carriers) to coexist within the $\mathrm{Cu}-\mathrm{O}$ plane, by promoting spatial segregation of holes into stripe-like patterns. Although evidence for the existence of such charge stripes have been obtained [23], it remains to be clarified whether these stripes would be conducive (or detrimental) to the formation of a superconducting ground state. Interestingly, it has recently been predicted theoretically that the existence of a striped phase in heavy-fermion systems is also a distinct possibility [24]. It was suggested that these so-called Kondo-stripes separate electronically inhomogeneous heavy Fermi liquid phases and result in a modulation of the charge density, an effect which should be visible experimentally. If verified, it would be interesting to see the manner in which heavy-fermion superconductivity is influenced by the existence of such charge density modulations.

In summary, we have discussed the implications of our magnetotransport data obtained in CeIrIn ${ }_{5}$ and their relation to prior observations on the high-temperature superconducting cuprates. Two of our key observations-a pseudogaplike precursor state and the existence of two distinct scattering times-are reminiscent of the behavior in the cuprates. These observations are also consistent with a scenario in which incipient antiferromagnetic fluctuations crucially influence the magnetotransport in these two disparate classes of systems by modifying the electron scattering rates along different parts of the Fermi surface. The phenomenon of unconventional superconductivity in the heavy-fermion systems, the high- $T_{\mathrm{c}}$ cuprates, and maybe even the newly discovered iron-oxypnictides can thus possibly be placed on a universal platform: the nature and properties of the electronic ground state are dictated by magnetic fluctuations.

Acknowledgements S.N. was supported by the A. von Humboldt Foundation. Work at Dresden was supported by the EC $(\mathrm{CoMePhS}$ 517039) and the DFG (Forschergruppe 960). Work at Los Alamos was performed under the auspices of the U.S. Department of Energy/Office of Science.
Open Access This article is distributed under the terms of the Creative Commons Attribution Noncommercial License which permits any noncommercial use, distribution, and reproduction in any medium, provided the original author(s) and source are credited.

\section{References}

1. Bardeen, J., Cooper, L.N., Schrieffer, J.R.: Phys. Rev. 108, 1175 (1957)

2. Steglich, F., Aarts, J., Bredl, C.D., Lieke, W., Meschede, D., Franz, W., Schäfer, H.: Phys. Rev. Lett. 43, 1892 (1979)

3. Mathur, N.D., Grosche, F.M., Julian, S.R., Walker, I.R., Freye, D.M., Haselwimmer, R.K.W., Lonzarich, G.G.: Nature 394, 39 (1998)

4. Schofield, A.J.: Contemp. Phys. 40, 95 (1999)

5. Sarrao, J.L., Thompson, J.D.: J. Phys. Soc. Jpn. 76, 051013 (2007)

6. Nakajima, Y., Shishido, H., Nakai, H., Shibauchi, T., Behnia, K., Izawa, K., Hedo, M., Uwatoko, Y., Matsumoto, T., Settai, R., Onuki, Y., Kontani, H., Matsuda, Y.: J. Phys. Soc. Jpn. 76, 024703 (2007)

7. Bel, R., Behnia, K., Nakajima, Y., Izawa, K., Matsuda, Y., Shishido, H., Settai, R., Onuki, Y.: Phys. Rev. Lett. 92, 217002 (2004)

8. Matsuda, Y., Izawa, K., Vekhter, I.: J. Phys.: Condens. Matter 18, R705 (2006)

9. Petrovic, C., Movshovich, R., Jaime, M., Pagliuso, P.G., Hundley, M.F., Sarrao, J.L., Fisk, Z., Thompson, J.D.: Europhys. Lett. 53, $354(2001)$

10. Paglione, J., Tanatar, M.A., Hawthorn, D.G., Boaknin, E., Hill, R.W., Ronning, F., Sutherland, M., Taillefer, L., Petrovic, C., Canfield, P.C.: Phys. Rev. Lett. 91, 246405 (2003)

11. Capan, C., Bianchi, A., Ronning, F., Lacerda, A., Thompson, J.D., Hundley, M.F., Pagliuso, P.G., Sarrao, J.L., Movshovich, R.: Phys. Rev. B. 70, 180502(R) (2004)

12. Nair, S., Wirth, S., Nicklas, M., Sarrao, J.L., Thompson, J.D., Fisk, Z., Steglich, F.: Phys. Rev. Lett. 100, 137003 (2008)

13. Chien, T.R., Wang, Z.Z., Ong, N.P.: Phys. Rev. Lett. 67, 2088 (1991)

14. Abe, Y., Segawa, K., Ando, Y.: Phys. Rev. B. 60, 15055(R) (1999)

15. Nakajima, Y., Shishido, H., Nakai, H., Shibauchi, T., Hedo, M., Uwatoko, Y., Matsumoto, T., Settai, R., Onuki, Y., Kontani, H., Matsuda, Y.: Phys. Rev. B 77, 214504 (2008)

16. Nair, S., Nicklas, M., Steglich, F., Sarrao, J.L., Thompson, J.D., Schofield, A.J., Wirth, S.: arXiv:0808.2377

17. Anderson, P.W.: Phys. Rev. Lett. 67, 2092 (1991)

18. Stojkovic, B.P., Pines, D.: Phys. Rev. B 55, 8576 (1997)

19. Tanatar, M.A., Paglione, J., Petrovic, C., Taillefer, L.: Science 316, 1320 (2007)

20. Zheng, G.-q., Tanabe, K., Mito, T., Kawasaki, S., Kitaoka, Y., Aoki, D., Haga, Y., Onuki, Y.: Phys. Rev. Lett. 86, 4664 (2001)

21. Kasahara, Y., Iwasawa, T., Shimizu, Y., Shishido, H., Shibauchi, T., Vekhter, I., Matsuda, Y.: Phys. Rev. Lett. 100, 207003 (2008)

22. Pham, L.D., Park, T., Maquilon, S., Thompson, J.D., Fisk, Z.: Phys. Rev. Lett. 97, 056404 (2006)

23. Tranquada, J.M., Sternlieb, B.J., Axe, J.D., Nakamura, Y., Uchida, S.: Nature 375, 561 (1995)

24. Zhu, J.-X., Martin, I., Bishop, A.R.: Phys. Rev. Lett. 100, 236403 (2008) 